\title{
Does the Viscosity Exponent Derive from Ultrasonic Attenuation Spectra?
}

\author{
J. K. Bhattacharjee · S. Z. Mirzaev • U. Kaatze
}

Received: 26 October 2011 / Accepted: 6 February 2012 / Published online: 29 February 2012 (C) The Author(s) 2012. This article is published with open access at Springerlink.com

\begin{abstract}
Based on a representation of the sound velocity of critical liquids in terms of a frequency-dependent complex specific heat at constant pressure, a simple relation between the low-frequency normalized sonic attenuation coefficient and the correlation length of fluctuations is derived. This relation provides a promising alternative for the determination of the dynamics exponent and thus the critical exponent of the shear viscosity. Sonic attenuation data from the literature, measured at frequencies down to $50 \mathrm{kHz}$, are re-evaluated with a view of the viscosity exponent determination. It is found that only in a small temperature range, the major requirement of the approach is fulfilled with the available data. Close to the critical temperature, the frequencies of measurement are still insufficiently small as compared to the inverse relaxation time of order parameter fluctuations. Criteria for future experiments are discussed briefly.
\end{abstract}

Keywords Binary mixtures $\cdot$ Critical shear viscosity $\cdot$ Scaling · Ultrasonic attenuation · Viscosity exponent

\footnotetext{
J. K. Bhattacharjee

S. N. Bose National Center for Basic Sciences, Salt Lake, Kolkata 700098, India e-mail: jayanta.bhattacharjee@gmail.com
}

\section{S. Z. Mirzaev}

Heat Physics Department, Uzbek Academy of Sciences, Katartal Street 28,

Tashkent 100135, Uzbekistan

e-mail: mirzaev@web.de

U. Kaatze $(\varangle)$

Drittes Physikalisches Institut, Georg-August-Universität, Friedrich-Hund-Platz 1, 37077 Göttingen, Germany

e-mail: uka@physik3.gwdg.de 


\section{Introduction}

In fluids near a critical point, the behavior of the systems is largely dominated by longrange fluctuations. Effectively masking the individual interactions of the fluids, critical fluctuations induce universal characteristics in thermodynamic and transport properties. Universality, involving scaling and power laws, has attracted much attention in the past decades and is still today a topic of lively scientific debate [1-3].

The exponent $x_{\eta}$ for the divergence of the critical shear viscosity has been elusive both experimentally and theoretically for quite some time. Being a small exponent, it is difficult to measure directly and likewise difficult to calculate. Early analyses of the mode-coupling equations lead to a first approximation $x_{\eta}=8 /\left(15 \pi^{2}\right)=0.054$ [4-7]. Later, vertex corrections resulted in $x_{\eta}=0.070 \pm 0.008$ [8] and renormalization-group theory [9] as well as mode-coupling theory [10] of the critical dynamics yielded $x_{\eta}=0.065$. The latter value is in fair agreement with $x_{\eta}=0.0635 \pm 0.0004$, as directly obtained from shear viscosity data [11,12], and with $x_{\eta}=0.063 \pm 0.024$ from extrapolation of the decay rate of order parameter fluctuations as derived from photon-correlation spectroscopy [13]. Space shuttle experiments, which allowed a closer approach to the critical point in the microgravity environment, determined the exponent as $x_{\eta}=0.0690 \pm 0.0006$ [14]. A three-loop mode-coupling calculation including the memory effect and vertex corrections yielded $x_{\eta}=0.0679 \pm 0.0007$ and provided a decent understanding [15].

The issue that we would like to address is whether there is a terrestrial indirect experiment which can be equally precise as the direct determination in a space shuttle. So far, it is the quasi-elastic light scattering experiments by Burstyn and Sengers [13] which provide the prime source for an alternative determination of the viscosity exponent. In this article, we investigate the low-frequency sound attenuation of binary liquids with a view of its suitability for a reliable viscosity exponent determination. Binary liquids are ideally suited for this purpose, because the specific heat in such mixtures is dominated by the large non-critical background contribution that exists at the consolute point.

\section{Theory}

The sound velocity $c_{\mathrm{S}}$ near the consolute point temperature $T_{\mathrm{c}}$ can be expressed by the formula [16],

$$
c_{\mathrm{s}}^{2}=c_{0}^{2}+c_{1}^{2} k_{\mathrm{B}} / C_{p},
$$

where $c_{0}$ is the non-critical part, $k_{\mathrm{B}}$ is Boltzmann's constant, and the specific heat $C_{p}$ is the quantity in Eq. 1 which shows critical behavior. In the thermodynamic limit at zero frequency $C_{p}$, according to the relation,

$$
C_{p}=C_{0} t^{-\alpha_{0}}+\tilde{C}_{p}=\Delta C+\tilde{C}_{p}
$$


diverges as the temperature $T$ approaches $T_{\mathrm{c}}$. Here $t=\left|T-T_{\mathrm{c}}\right| / T_{\mathrm{c}}$ is the scaled (reduced) temperature, $\tilde{C}_{p}$ is the large background part, and $\Delta C=C_{0} t^{-\alpha_{0}}$ is the critical term with critical exponent $\alpha_{0}(=0.11$ [17]). Equation 2 follows from a more comprehensive heat-capacity formulation $[18,19]$, if a possible small temperature dependence in the background part is neglected. The small exponent for heat capacity ensures that for $t$ larger than about $10^{-4}$, the non-critical background part $\tilde{C}_{p}$ dominates $C_{p}$. A passing sound wave of frequency $v$ causes the temperature to oscillate with the same frequency, and the entropy responding to the temperature oscillation gives rise to a frequency-dependent specific heat. In general, the entropy will exhibit a phase lag relative to the temperature, so that a complex specific heat follows. For that reason, frequency enters through the combination $-\mathrm{i} \omega=-\mathrm{i} 2 \pi \nu$ in the equations $\left(\mathrm{i}^{2}=-1\right)$. Hence, the specific heat depends upon frequency and temperature and adopts the scaling form

$$
C_{p}=\Delta C(\omega, t)+\tilde{C}_{p}=C_{0} t^{-\alpha_{0}} f\left(-\mathrm{i} \omega \xi^{Z}\right)+\tilde{C}_{p}=C_{0} \xi_{0}^{-\alpha_{0} / \tilde{v}^{2}} \xi^{\alpha_{0} / \tilde{v}} f\left(-\mathrm{i} \omega \xi^{Z}\right)+\tilde{C}_{p}
$$

In this equation,

$$
\xi=\xi_{0} t^{-\tilde{v}}
$$

is the correlation length, which diverges as $T_{\mathrm{c}}$ is approached with the critical exponent $\tilde{v}(=0.63[20])$, and

$$
Z=D+x_{\eta}
$$

where $D$ denotes the dimensionality of space, and $Z$ is the dynamics critical exponent. Equation 3 expresses the critical slowing down of the system as it seeks to re-gain thermal equilibrium after a small disturbance. The larger the mean sizes $\xi$ of correlated regions, the longer the correlations take to decay and the decay time,

$$
\tau=\left(3 \pi \eta_{\mathrm{b}} \lambda_{\mathrm{D}} x_{\eta} \xi^{Z}\right) /\left(k_{\mathrm{B}} T\right)
$$

diverges as $\tau \sim \xi^{Z}$. Here $\eta_{\mathrm{b}}$ is the non-critical background viscosity and $\lambda_{\mathrm{D}}$ is a Debye cut-off length. Hence, Eq. 3 expresses the natural fact that the specific heat is a function of $\omega \tau$. For binary mixtures $D=3$, so that the exponent $x_{\eta}$, which we wish to determine, is small compared to $Z$.

Having introduced the idea of a complex specific heat, it is now a straightforward matter to obtain the critical sound attenuation as a function of frequency and temperature. It derives from the sound velocity which, induced by the complex specific heat, is complex. From Eq. 1 we get

$$
\begin{aligned}
c_{\mathrm{s}}^{2} & =c_{0}^{2}+c_{1}^{2} \frac{k_{\mathrm{B}}}{\Delta C(\omega, \xi)+\tilde{C}_{p}} \\
& =c_{0}^{2}+c_{1}^{2} \frac{k_{\mathrm{B}}}{\operatorname{Re} \Delta C(\omega, \xi)+\operatorname{iIm} \Delta C(\omega, \xi)+\tilde{C}_{p}}
\end{aligned}
$$




$$
\begin{aligned}
& =c_{0}^{2}-\mathrm{i} \frac{c_{1}^{2} k_{\mathrm{B}} \operatorname{Im} \Delta C(\omega, \xi)}{\left[\operatorname{Re} \Delta C(\omega, \xi)+\tilde{C}_{p}\right]^{2}+[\operatorname{Im} \Delta C(\omega, \xi)]^{2}} \\
& \approx c_{0}^{2}-\mathrm{i} \frac{c_{1}^{2} k_{\mathrm{B}} \operatorname{Im} \Delta C(\omega, \xi)}{\tilde{C}_{p}^{2}}
\end{aligned}
$$

The complex wave number $k$ of a sound wave with frequency $v$ follows from

$$
k^{2}=\omega^{2} / c_{\mathrm{s}}^{2} \approx \omega^{2} / c_{0}^{2}\left[1+\mathrm{i}\left(c_{1}^{2} / c_{0}^{2}\right) k_{\mathrm{B}} \operatorname{Im} \Delta C(\omega, \xi) / \tilde{C}_{p}^{2}\right] .
$$

With notation $k=k_{1}+\mathrm{i} k_{2}$ and because $k_{2} \ll k_{1}$ we have

$$
k^{2}=\left(k_{1}+\mathrm{i} k_{2}\right)^{2} \approx k_{1}^{2}+2 \mathrm{i} k_{1} k_{2}=k_{1}^{2}\left[1+2 \mathrm{i} \frac{k_{2}}{k_{1}}\right] .
$$

Hence, the critical attenuation per wavelength,

$$
\alpha_{\lambda}^{\mathrm{c}}(\nu, T)=\alpha \lambda(\nu, T)-(\alpha \lambda)_{\mathrm{bg}}(\nu, T)
$$

is given by

$$
\alpha_{\lambda}^{\mathrm{c}}=\frac{k_{1}}{k_{2}}=\frac{c_{1}^{2}}{2 c_{0}^{2}} k_{\mathrm{B}} \frac{\operatorname{Im} \Delta C}{\tilde{C}_{p}^{2}} .
$$

Let us now focus on the scaling form of $\Delta C$. From Eq.3, we get

$$
\Delta C=C_{0} \xi^{\alpha_{0} / \tilde{v}} f\left(-\mathrm{i} \omega \xi^{Z}\right),
$$

with $f(0)=1$. At fixed $\omega$ and $\omega<\tau^{-1}$, the response of the system increases as $\xi^{\alpha_{0} / \tilde{v}}$ and stops to increase when $\omega \approx \tau^{-1}$ as the system can no longer follow the high-frequency oscillations. For $\omega>\tau^{-1}$, the value at $\omega \approx \tau^{-1}$ is retained, and thus for high frequencies

$$
\Delta C\left(\omega>\tau^{-1}\right) \approx \tilde{C}_{0}(-\mathrm{i} \omega)^{-\delta},
$$

i.e., $f(x) \sim x^{-\delta}$ for $x \ll 1$, where $\delta=\alpha_{0} /(Z \tilde{v})$. Introducing the complex function $f(x)=f_{1}(x)+\mathrm{i} f_{2}(x)$, we get from Eqs. 11 and 13

$$
\frac{\alpha_{\lambda}^{\mathrm{c}}(\nu, T)}{\alpha_{\lambda}^{\mathrm{c}}\left(\nu, T_{\mathrm{c}}\right)}=\left(\omega \xi^{Z}\right)^{\delta} f_{2}\left(\omega \xi^{Z}\right)=g\left(\omega \xi^{Z}\right) .
$$

At this point, a choice has to be made about the analytic properties of $f_{2}(x)$ and $g(x)$. If $f_{2}(x)$ is analytic and proportional to $x$ at $x \ll 1$, then the ratio on the left-hand side of Eq. 14 is proportional to $x^{1+\delta}=x^{1+\alpha_{0} /(Z \tilde{v})}$ for $x \ll 1$, whereas the analytic behavior of $g(x)$ at $x \ll 1$ means that the attenuation-per-wavelength ratio is proportional to $x$. The choice is to be made by experimental data. 
We also see that the normalized low-frequency sonic attenuation data scale as $\xi^{Z(1+\delta)}$ or $\xi^{Z}$, respectively, so that the dynamic scaling coefficient and thus the viscosity exponent may be obtained from suitable $\alpha_{\lambda}^{\mathrm{c}}$ determinations.

\section{Experimental Evidence}

\subsection{Ultrasonic Attenuation Spectra}

A careful inspection of the literature revealed two papers by Lisnyanskii et al. [21,22] in which ultrasonic attenuation coefficients for the nitrobenzene- $n$-hexane mixture of critical composition are reported at frequencies down to $50 \mathrm{kHz}$. Adiabatic compressibility measurements of the same critical system have indeed been conducted down to $18 \mathrm{~Hz}$ [23]. To the best of our knowledge, however, no attenuation data for any binary critical liquid are available at frequencies below $50 \mathrm{kHz}$. We even do not know other binary systems for which sonic attenuation data down to $50 \mathrm{kHz}$ have been reported and for which, in addition, the other parameters, required in this analysis, have been determined. For that reason, we shall focus on the nitrobenzene- $n$-hexane system [21,22] and shall compare the results to the more recently investigated binary $n$-pentanol-nitromethane critical mixture [24]. The latter binary system had indeed been measured down to $180 \mathrm{kHz}$ only. In our analysis, however, the adverse effect of a higher low-frequency limit of the sonic attenuation data will be partly compensated by the extremely small amplitudes [3] of the relaxation time $\left(\tau_{0}=5.3 \mathrm{ps}\right)$ and fluctuation correlation length $\left(\xi_{0}=0.15 \mathrm{~nm}\right)$.

Both systems exhibit an upper critical demixing point with a convenient critical temperature: $T_{\mathrm{c}}=293.35 \mathrm{~K}, X_{\mathrm{c}}=0.40$, nitrobenzene- $n$-hexane [21,22]; $T_{\mathrm{c}}=$ $300.95 \mathrm{~K}, X_{\mathrm{c}}=0.385, n$-pentanol-nitromethane; $X_{\mathrm{c}}=$ mole fraction of the first constituent, respectively. Unfortunately, the $T_{\mathrm{c}}$ values reported in other sonic attenuation studies of the former binary critical mixture vary noticeably around the above value (293.13 K $\leq T_{\mathrm{c}} \leq 294.15 \mathrm{~K}$ [23,25-29]).

The low-frequency sonic attenuation data of the nitrobenzene- $n$-hexane critical mixture had been measured with the aid of a statistical reverberation method [22], for which uncertainties $\Delta \alpha / \alpha=0.1(0.05 \mathrm{MHz} \leq v \leq 0.1 \mathrm{MHz})$ and $\Delta \alpha / \alpha=$ $0.07(0.1 \mathrm{MHz} \leq v \leq 1 \mathrm{MHz})$ had been given [30]. The low-frequency $n$-pentanolnitromethane data were obtained from cavity resonator measurements [24], using a plano-concave cylindrical cell to reduce disturbances from mechanical stress at varying temperature [31]. The uncertainty was $\Delta \alpha / \alpha=0.05$ in the range $0.18 \mathrm{MHz} \leq$ $v \leq 1 \mathrm{MHz}$. It is worthwhile to note, however, that attenuation coefficient ratios (Eq. 4) will be evaluated for the viscosity exponent determination. Forming the ratios, part of the experimental errors will compensate.

In Fig. 1, an ultrasonic attenuation spectrum for the nitrobenzene- $n$-hexane mixture of critical composition at $303.15 \mathrm{~K}$ is displayed in both common formats. In these plots, data at frequencies between $71 \mathrm{kHz}$ and $158 \mathrm{MHz}$ [21] are supplemented by such between $24 \mathrm{MHz}$ and $1 \mathrm{GHz}$ [26]. In the frequency-normalized format, the $\alpha / v^{2}$ data decrease monotonically with frequency $v$ to approach a limiting high-frequency value 
$B^{\prime}$. Obviously, however, this decrease in $\alpha / v^{2}$ cannot be adequately represented by a Bhattacharjee-Ferrell relaxation function ("BF" $[3,32]$ ),

$$
R_{\mathrm{BF}}^{\prime}(v)=A_{\mathrm{BF}}^{\prime}(T) v^{-(1-\delta)} F_{\mathrm{BF}}(v)
$$

with frequency-independent amplitude $A_{\mathrm{BF}}^{\prime}$ and scaling function,

$$
\begin{aligned}
F_{\mathrm{BF}}(v) & =\left[1+0.414\left\{\Omega_{1 / 2} /(\omega \tau)\right\}^{1 / 2}\right]^{-2} \\
& =\left[1+0.414\left\{\Omega_{1 / 2} / \Omega\right\}^{1 / 2}\right]^{-2} \\
& =\left[1+0.6 \Omega^{1 / 2}\right]^{-2}
\end{aligned}
$$

where $\Omega_{1 / 2}(=2.1)$ denotes a scaled half-attenuation frequency and $\Omega=2 \pi \nu \tau$. Rather, an additional Debye relaxation term [33],

$$
R_{\mathrm{D}}^{\prime}(v)=A_{\mathrm{D}}^{\prime}\left[1+\left(\omega \tau_{\mathrm{D}}\right)^{2}\right]^{-1}
$$

is required to represent the experimental data within their limits of error (Fig. 1). In Eq. $17, A_{\mathrm{D}}^{\prime}$ is the relaxation amplitude and $\tau_{\mathrm{D}}$ is the relaxation time. Failing to notice the Debye-type contribution to the sonic attenuation spectra has lead to scaling functions in the critical contribution which did not satisfy the limiting condition of the scaling function to asymptotically approach 1 at high frequencies [21,26,28]. However, taking the Debye term into account, thus representing the experimental $\alpha / v^{2}$ data by the spectral function,

$$
R^{\prime}(v)=R_{\mathrm{BF}}^{\prime}(v)+R_{\mathrm{D}}^{\prime}(v)+B^{\prime}
$$

results in a favorable representation of data ([34], Fig. 1). In correspondence with Eq. 18 the excess attenuation per wavelength,

$$
(\alpha \lambda)_{\mathrm{exc}}=\left(\alpha / v^{2}\right) c_{\mathrm{s}} v-B^{\prime} c_{\mathrm{s}} v
$$

has been represented by the sum of a critical term $R_{\mathrm{BF}}=R_{\mathrm{BF}}^{\prime} c_{\mathrm{s}} v$ and a Debye term $R_{\mathrm{D}}=R_{\mathrm{D}}^{\prime} c_{\mathrm{s}} v$. The need for a relaxation term, in addition to the $\mathrm{BF}$ term, is also clearly indicated by the $(\alpha \lambda)_{\text {exc }}$ spectrum in the inset of Fig. 1 .

In Fig. 2, the frequency normalized attenuation spectrum of the $n$-pentanol-nitromethane mixture of critical composition is displayed at $307.95 \mathrm{~K}$. The data do not reveal a Debye-type relaxation. Hence, the experimental spectra have been analytically represented by a sum of a BF term and the frequency-independent $B^{\prime}$ term [24]. The inset shows the low-frequency data which are the focus of interest here. 


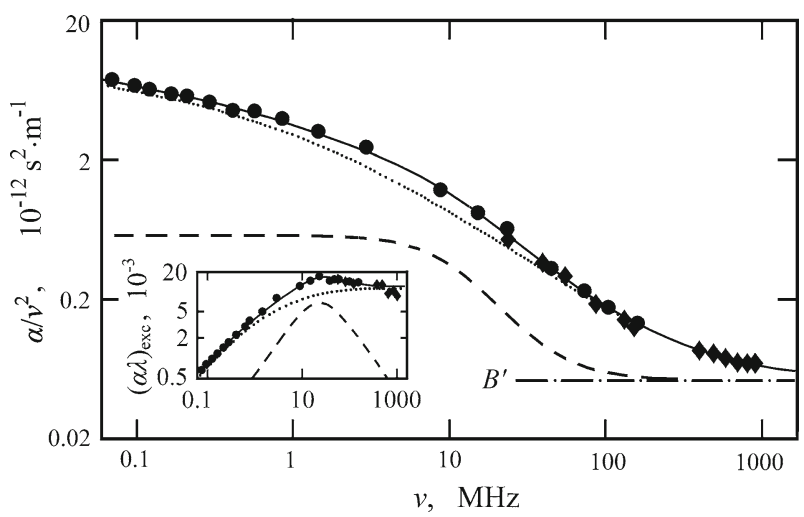

Fig. 1 Frequency-normalized sonic attenuation coefficient $\alpha / v^{2}$ displayed as a function of frequency $v$ for the nitrobenzene- $n$-hexane mixture of critical composition at $30{ }^{\circ} \mathrm{C}(\bullet[21], \diamond[25])$. Inset shows the same data in the excess-attenuation-per-wavelength format. Dotted lines are graphs of the Bhattacharjee-Ferrell relaxation function for critical systems (Eq. 15), dashed lines indicate a Debye relaxation term (Eq. 17), and dashed-dotted line represents the asymptotic high-frequency contribution. The sum of the individual contributions is shown by full lines

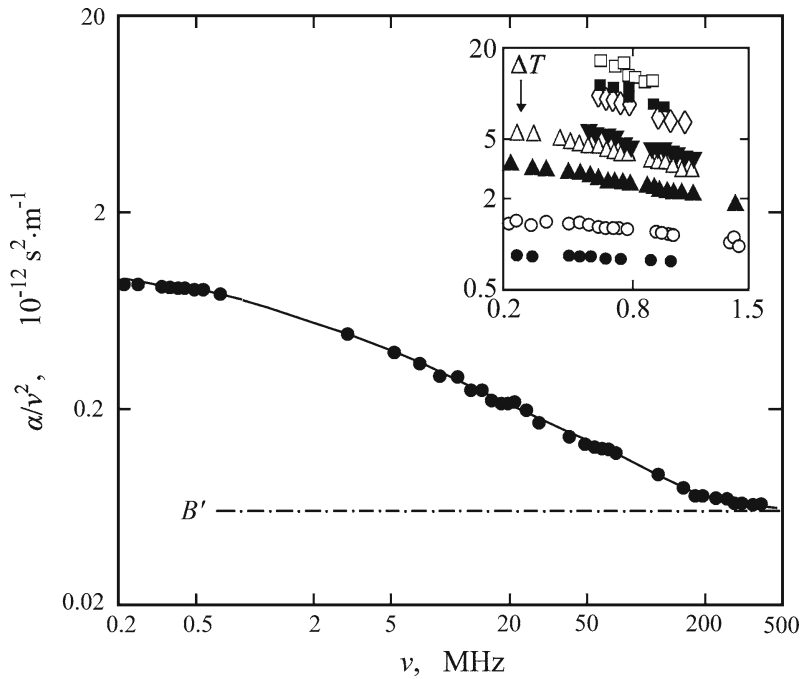

Fig. 2 Frequency-normalized sonic attenuation spectrum for the $n$-pentanol-nitromethane mixture of critical composition at $307.95 \mathrm{~K}$. Dashed-dotted line shows the asymptotic high-frequency contribution $B^{\prime}$, full line represents the sum of this contribution and of the Bhattacharjee-Ferrell relaxation function (Eq. 15). In the inset, data from a run of plano-concave resonator measurements are displayed at a set of temperatures: $\square 300.95 \mathrm{~K}, \square 301.39 \mathrm{~K}, \diamond 301.68 \mathrm{~K}, \nabla 302.55 \mathrm{~K}, \Delta 303.01 \mathrm{~K}, \boldsymbol{\Delta} 303.96 \mathrm{~K}$, 。 $305.91 \mathrm{~K}, \bullet 307.84 \mathrm{~K}$

\subsection{Scaling Function}

The critical contribution to the attenuation per wavelength (Eq. 10) follows as

$$
\alpha_{\lambda}^{\mathrm{c}}(v, T)=\left(\alpha / v^{2}\right) c_{\mathrm{S}} v-R_{\mathrm{D}}(v, T)-B(T) v
$$


where $B=B^{\prime} c_{\mathrm{S}}$. As we are interested in the low-frequency part of the spectra $(v \leq$ $3 \mathrm{MHz}$ ), where $R_{\mathrm{D}}^{\prime}$ is independent of frequency and, thus $R_{\mathrm{D}}(v)=A_{\mathrm{D}} v$, we simply use

$$
\alpha_{\lambda}^{\mathrm{c}}(v, T)=\left(\alpha / v^{2}\right) c_{\mathrm{s}} v-B^{*}(T) v=\left(\alpha / v^{2}\right) c_{\mathrm{s}} v-\left[B(T)+A_{\mathrm{D}}(T)\right] v
$$

in the evaluation of data. Extrapolating at every frequency of measurement $\alpha_{\lambda}^{\mathrm{c}}(\nu, T)$ for a small temperature difference to obtain the critical contribution $\alpha_{\lambda}^{\mathrm{c}}\left(v, T_{\mathrm{c}}\right)$ at the critical temperature, the low-frequency scaling function data have been calculated as the ratio, $F(v, T)=\alpha_{\lambda}^{\mathrm{c}}(v, T) / \alpha_{\lambda}^{\mathrm{c}}\left(v, T_{\mathrm{c}}\right)$. In Fig. 3, for both critical systems, results from measurements at different temperatures $[21,24]$ are shown as a function of reduced frequency $\Omega$. The $\Omega$ values for the nitrobenzene- $n$-hexane critical mixture have been obtained using the power law,

$$
\tau(T)=\tau_{0} t^{-Z \tilde{v}}
$$

and $\tau_{0}=23 \mathrm{ps}[34]$ resulting from an analogous application of Eq. 6

$$
\tau_{0}=\left(3 \pi \eta_{\mathrm{b}} \lambda_{\mathrm{D}} x_{\eta} \xi_{0}^{Z}\right) /\left(k_{\mathrm{B}} T\right)
$$

if literature values $\xi_{0}=0.265 \mathrm{~nm}[35,36]$ and $\eta_{\mathrm{b}} \lambda_{\mathrm{D}} x_{\eta}=5.5 \times 10^{-6} \mathrm{~Pa} \cdot \mathrm{s}$ [37] are inserted. This relaxation time amplitude compares to $\tau_{0}=18 \mathrm{ps}$ which is obtained from a regression analysis of the experimental $\alpha_{\lambda}^{\mathrm{c}}(\nu, T) / \alpha_{\lambda}^{\mathrm{c}}\left(\nu, T_{\mathrm{c}}\right)$ data in terms of the BF scaling function (Eq. 16), treating $\tau_{0}$ as the only adjustable parameter. With the $n$-pentanol-nitromethane mixture of critical composition, the relaxation times $\tau(T)$, and thus the reduced frequencies $\Omega$, were obtained from a combined evaluation of shear viscosity and quasi-elastic light scattering data [24], taking effects of crossover from singular to mean-field behavior into account [38].

At $\Omega<0.02$, the normalized attenuation-per-wavelength values in Fig. 3 display a linear dependence upon $\Omega$. A small curvature becomes evident, however, at larger reduced frequencies. As indicated by the data for the critical system nitroethanecyclohexane (Fig. 3, inset), the linear range in the $\alpha_{\lambda}^{\mathrm{c}}(\nu, T) / \alpha_{\lambda}^{\mathrm{c}}\left(\nu, T_{\mathrm{c}}\right)$ versus $\Omega$ plot becomes noticeably smaller if, at an almost identical low-frequency limit of measurements $(160 \mathrm{kHz})$, the amplitudes of the relaxation time $\left(\tau_{0}=6.4 \mathrm{ps}[39]\right)$ and fluctuation correlation length $\left(\xi_{0}=0.16 \mathrm{~nm}\right.$ [39]) are slightly larger than those of the $n$-pentanol-nitromethane mixture.

Because of the small range of linearity, it is difficult to conclude whether the normalized $\alpha_{\lambda}^{\mathrm{c}}$ data are proportional to $\Omega$ or rather to $\Omega^{(1+\delta)}$, i.e., it is hardly possible to sort out the analytic properties of the functions $f_{2}$ and $g$ in Eq. 14. We therefore have extended the range of linearity for the nitrobenzene- $n$-hexane and $n$-pentanol-nitromethane data by considering the function,

$$
F^{*}(v, T)=F(v, T)\left[0.6+(\omega \tau)^{1 / 2}\right]^{2},
$$




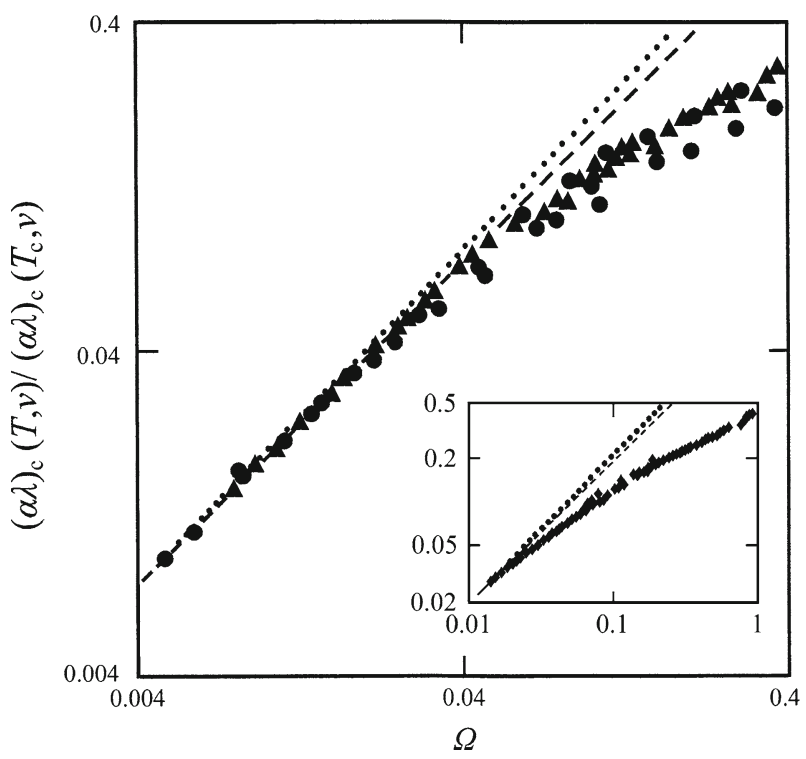

Fig. 3 Low-frequency part of the scaling function data versus reduced frequency $\Omega$ for the nitrobenzene$n$-hexane $\left(\bullet[34], \tau_{0}=23 \mathrm{ps}\right), n$-pentanol-nitromethane $(\boldsymbol{\Delta}$ [24]), as well as nitroethane-cyclohexane ( [39]) mixtures of critical composition,. The dashed and dotted lines indicate power law behavior with exponents 1 and $1+\delta$, respectively

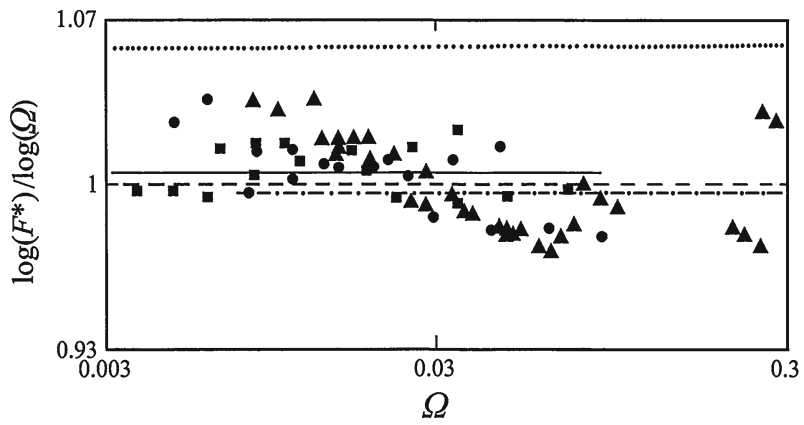

Fig. 4 Ratio of logarithms of corrected scaling function data $F^{*}$ (Eq. 24) and of $\Omega$, presented as a function of reduced frequency $\Omega$, for the nitrobenzene- $n$-hexane $\left(\bullet \tau_{0}=23 \mathrm{ps}, \tau_{0}=18 \mathrm{ps}\right)$, and $n$-pentanolnitromethane $(\boldsymbol{\Delta})$ mixtures of critical composition. Full line represents the arithmetic mean (1.005) of the former data, and dashed-dotted line that (0.996) of the latter data. Dashed and dotted lines indicate power law behavior (Eq. 14) with exponents 1 and 1.058, respectively

in which a correction for the slight curvature in the normalized attenuation-per-wavelength data has been made according to the frequency behavior of the theoretical scaling function (Eq. 16). In Fig. 4, the ratio $\sigma=\log \left(F^{*}\right) / \log (\Omega)$ at small $\Omega$ is shown for both critical systems. The mean values $\bar{\sigma}=1.005 \pm 0.025$ and $\bar{\sigma}=0.996 \pm 0.025$ result for the data of the nitrobenzene- $n$-hexane and $n$-pentanol-nitromethane systems, respectively. These means close to unity indicate that the exponent is 1 rather than $1+\delta=1.058$. Hence, the function $g$ in Eq. 16 is analytic. 


\subsection{Viscosity Exponent}

According to this result, we are now looking for the proportionality

$$
v^{-1} F(v, T)=\chi \xi^{Z}(T)
$$

between the frequency-normalized attenuation coefficient ratio and $\xi^{Z}$, as predicted by Eq. 14. Here $\chi$ is a factor. Hence, unlike the scaling function plot of Fig. 3, we now want to ignore the frequency dependence in the $F(v, T)$ data to focus on their dependence upon the correlation length (and thus temperature). For this purpose, we resort to low-frequency sonic attenuation data between $293.55 \mathrm{~K}$ and $308.15 \mathrm{~K}$ from both papers by Lisnyanskii et al. [21,22] as well as between $300.95 \mathrm{~K}$ and $307.84 \mathrm{~K}$ from the paper by Iwanowski et al. [24]. In Figs. 5 and 6 the $v^{-1} F$ data for both systems are displayed at all temperatures $T$ of measurement as a function of $v$. Only at temperatures $T=T_{\mathrm{c}}+\Delta T$ far from $T_{\mathrm{c}}$, the $v^{-1} F$ values are independent of frequency ( $\Delta T=14.8 \mathrm{~K}$, nitrobenzene- $n$-hexane; $\Delta T=6.9 \mathrm{~K}, n$-pentanol-nitromethane). Closer to the critical temperature, the experimental data evidently do not correspond with the strictly linear range. We thus have determined the desired frequency-independent limiting values by extrapolation:

$$
\lim _{v \rightarrow 0} v^{-1} F(v)=C^{-2},
$$

assuming the frequency dependence

$$
v^{-1} F(v)=\left(C+v^{1 / 2}\right)^{-2}
$$

Fig. 5 Frequency-normalized scaling function data versus frequency $v$ for the nitrobenzene- $n$-hexane mixture of critical composition at various temperatures $T=T_{\mathrm{c}}+\Delta T$. Circles show the limiting data as obtained from extrapolation according to Eqs. 26 and 27

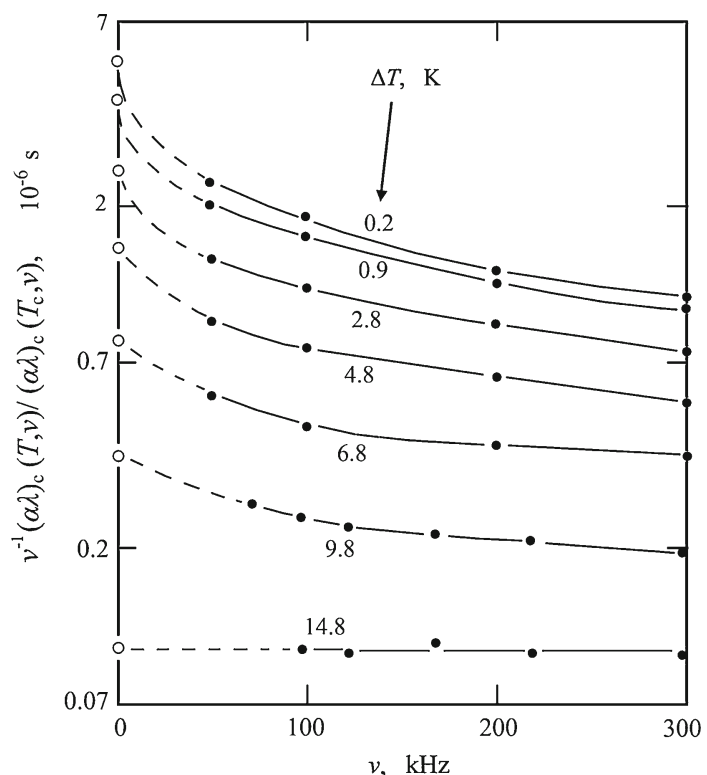


Fig. 6 Frequency-normalized scaling function data (full symbols) versus frequency $v$ for the $n$-pentanol-nitromethane mixture of critical composition at some temperature differences $\Delta T$ to the critical temperature. Open symbols show the limiting data as obtained from extrapolation according to Eqs. 26 and 27

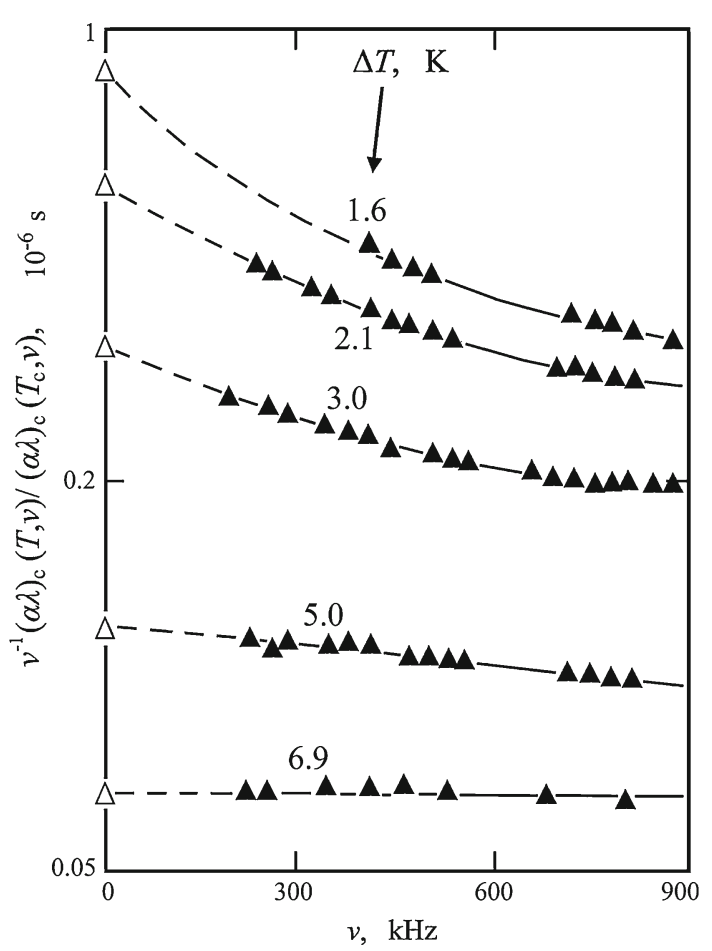

of the BF scaling function (Eq. 16). The resulting $C$ values are shown by circles and open triangles in Figs. 5 and 6, respectively, and are plotted versus the correlation length $\xi$ in Fig. 7. Additionally given for the nitrobenzene- $n$-hexane system are $v^{-1} \tilde{F}(v)$ values in which corrections for temperature effects in the proportionality factor $\chi$ (Eq. 25) have been implied. Considering the BF scaling function (Eq. 16) at $\Omega \ll 0.36$,

$$
v^{-1} F(\nu, T)=2 \pi \tau=\left[6 \pi^{2} \eta_{\mathrm{b}}(T) \lambda_{\mathrm{D}} x_{\eta}\right] \xi^{Z} /\left(k_{\mathrm{B}} T\right)
$$

indicating that $\chi$ depends upon temperature due to the $T$-dependence in the background viscosity $\eta_{\mathrm{b}}$ and to the temperature in the denominator on the right-hand side of Eq. 28. Using

$$
\eta_{\mathrm{b}}(T)=A \exp (B / T),
$$

with $B=1.05 \times 10^{3} \mathrm{~K}[37,40]$, the original $F$ data of the nitrobenzene- $n$-hexane mixture have been corrected relative to the reference values at $35^{\circ} \mathrm{C}(\Delta T=14.8 \mathrm{~K})$ to yield

$$
\tilde{F}(\nu, T)=F(\nu, T) \eta_{\mathrm{b}, \text { ref }} T /\left(\eta_{\mathrm{b}} T_{\text {ref }}\right) .
$$


Fig. 7 Extrapolated frequency-normalized scaling function data from Figs. 5 (o) and $6(\boldsymbol{\Delta})$ as well as nitrobenzene- $n$-hexane data corrected according to Eq. 30 $(\bullet)$, plotted as a function of the correlation length $\xi$ corresponding with the temperature difference $\Delta T=T-T_{\mathrm{c}}$. Full line is shown to guide the eye. Dashed lines represent power law behavior with exponent $Z=3.069$

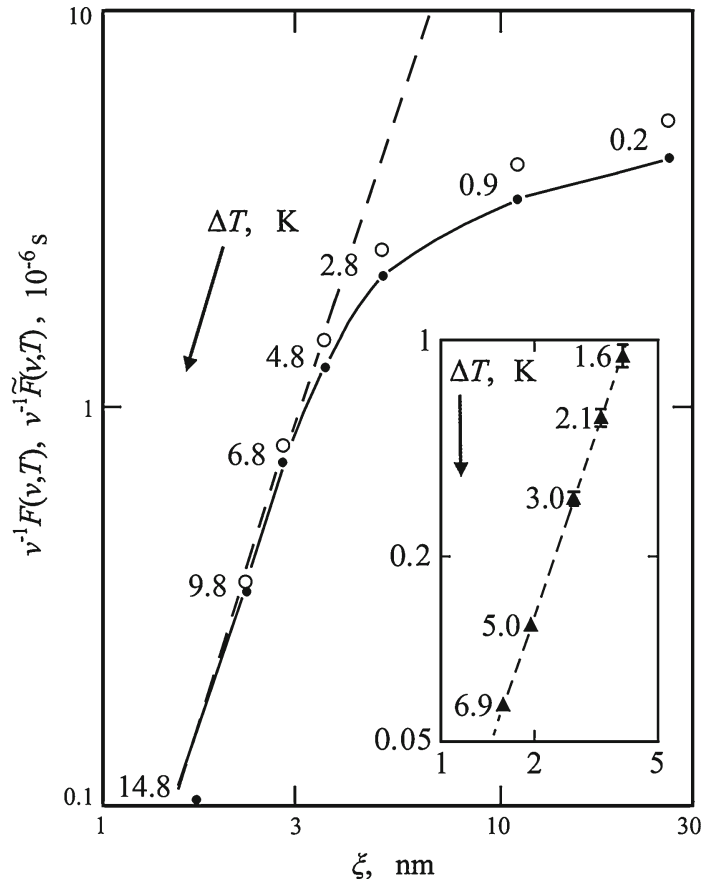

Obviously, the function $v^{-1} \tilde{F}(v, T)$, like $v^{-1} F(v, T)$ itself, is proportional to $\xi^{Z}$ only in a small temperature range. Deviations from the theoretically predicted behavior will be discussed in the next section.

\section{Discussion and Conclusions}

Close to the critical temperature $\left(\Delta T=T-T_{\mathrm{c}}<4 \mathrm{~K}\right.$, e.g., $\left.t<0.014\right)$, the experimental $F$ and $\tilde{F}$ values, respectively, deviate substantially from the anticipated power law behavior (Fig. 7). This is undoubtedly a reflection of the fact that the major requirement of the above theoretical treatment is not fulfilled here. Theory requires the (angular) frequency in the sonic attenuation coefficient determination to be small compared to the inverse relaxation time of order parameter fluctuations: $\omega(=2 \pi \nu) \ll 1 / \tau$. For the critical nitrobenzene- $n$-hexane system, however, the inverse relaxation time is as small as $1 /(2 \pi \tau)=5.3 \times 10^{3} \mathrm{~s}^{-1}$ at $\Delta T=0.2 \mathrm{~K}\left(t=6.8 \times 10^{-4}\right)$ and $1 /(2 \pi \tau)=$ $8.6 \times 10^{5} \mathrm{~s}^{-1}$ at $\Delta T=2.8 \mathrm{~K}\left(t=9.5 \times 10^{-3}\right)$. For the $n$-pentanol-nitromethane binary mixture it is $1 /(2 \pi \tau)=1.2 \times 10^{6} \mathrm{~s}^{-1}$ at $\Delta T=1.6 \mathrm{~K}\left(t=5.3 \times 10^{-3}\right)$. Obviously, for data measured at frequencies between $50 \mathrm{kHz}$ and $300 \mathrm{kHz}$ as well as $180 \mathrm{kHz}$ and $1000 \mathrm{kHz}$, respectively, the corrections for nonlinearity, as applied in the calculation of $\tilde{F}$ values, are extensively insufficient. Measurements at even lower frequencies are required near $T_{\mathrm{c}}$. Such measurements are difficult to perform with adequate accuracy as will be briefly outlined below. 
The low-frequency measurements of the nitrobenzene- $n$-hexane critical mixture discussed in this article were obtained applying a statistical reverberation method [30]. The sample cell was most carefully designed to minimize parasitic losses which might exceed the desired liquid losses. Wall losses were reduced by a small surfaceto-volume ratio and by use of thin walls made from acoustically compliant material. Sound radiation into the medium surrounding the liquid-filled cell was largely avoided by placing it into a vacuum chamber during measurements. Sound attenuation due to losses in the piezoelectric transducers and in the supporting assembly was kept low by design features, and attention was also directed to losses due to viscous waves in the boundary layers at the cell walls. These features required a liquid volume of $500 \mathrm{~cm}^{3}$ to $700 \mathrm{~cm}^{3}$ for the measurements down to $50 \mathrm{kHz}$ [30]. Evidently, the temperature of such a large liquid volume is difficult to control with necessary precision because, on the one hand, cell wall vibrations should not be restrained by a thermostatic jacket and, on the other hand, stirring should not be considered to prevent disturbances of the sound field.

Analogous restrictions apply for resonator techniques [41] which, on first glance, may be considered superior alternatives. Because of the large wavelength $\lambda$ of the sonic field within the liquid, e.g., $\lambda=24 \mathrm{~mm}$ for the nitrobenzene- $n$-hexane mixture of critical composition near $T_{\mathrm{c}}$, difficult to temperature-stabilize large sample volumes are also required in these techniques. For commonly applied cylindrically shaped cavity resonators with one-dimensional wave propagation, the lateral dimensions need to be sufficiently large to avoid intolerable masking of the desired liquid attenuation by diffraction losses. Diffraction can indeed be reduced, but not completely eliminated, by the use of concavely shaped transducers [31]. With respect to undesired diffraction losses, spherical resonators are in favor. In correspondence with cells for statistical reverberation measurements, however, their temperature can hardly be kept constant with the precision required in measurements of critical systems [42].

The so-called resonance reverberation method [43] has been applied in the frequency range between $20 \mathrm{kHz}$ and $2 \mathrm{MHz}$ to measure the sonic attenuation of ternary liquid mixtures near critical points $[44,45]$. The liquid volume was $70 \mathrm{~cm}^{3}$ in these measurements, which compares to the $80 \mathrm{~cm}^{3}$ volume required in the plano-concave resonator technique [31]. In contrast to the rather massive and firm walls of planoconcave resonators in use $[31,41]$, the walls of the resonance reverberation sample cells were made of thin fluoroplastic films [43]. Unfortunately, no information about the temperature control and the temperature stability of the sample liquids was given for the resonance reverberation method [43-45].

Since a significant extension of the range of measurements to lower frequencies $v$ is difficult, the alternative to fulfil the basic condition $v \ll(2 \pi \tau)^{-1}$ involves measurements at temperatures away from the critical where, according to Eq. 22, the relaxation time $\tau$ is smaller. The disadvantage in such a procedure is the reduction of the critical part in the sonic attenuation coefficient which may lead to a reduction in the accuracy in $\alpha_{\lambda}^{\mathrm{c}}$. Because of the unavoidable Stokes damping in liquids $[3,46]$, the critical part always competes with a non-critical background part and apart from $T_{\mathrm{c}}$ has thus to be obtained as a small difference between two large values. This may be the reason for the deviation of the experimental value from the proportional-to- $\xi^{Z}$ characteristics for the nitrobenzene- $n$-hexane system at $\Delta T=14.8 \mathrm{~K}$ in Fig. 7 . Another reason may 
be the need for corrections for the crossover from singular to mean-field behavior at temperatures distant from $T_{\mathrm{c}}$.

Having in mind, however, that so far investigations of critical systems have been performed with a view to the behavior near the critical point, particular attention to the optimum temperature range for determination of the viscosity exponent may lead to superior $Z$ values. Re-measurement of binary critical systems such as $n$-pentanolnitromethane with an extraordinarily small amplitude of their relaxation time might be a promising approach if the resonance reverberation technique, offering a favorable low-frequency limit at comparably small sample volume, is included. Measurements at frequencies below $180 \mathrm{kHz}$ will greatly enhance the reliability of the extrapolated $v^{-1}(\alpha \lambda)_{\mathrm{c}}(T, v) /(\alpha \lambda)_{\mathrm{c}}\left(T_{\mathrm{c}}, v\right)$ data (Fig. 6) and will thus reduce the uncertainty in the slope of the $v^{-1} F(v, T)$ versus $\xi$ relation (Fig. 7) significantly.

Acknowledgment S. Z. Mirzaev gratefully acknowledges financial support by the German Academic Exchange Service (DAAD), Bonn, Germany.

Open Access This article is distributed under the terms of the Creative Commons Attribution License which permits any use, distribution, and reproduction in any medium, provided the original author(s) and the source are credited.

\section{References}

1. A. Onuki, Phase Transition Dynamics (Cambridge University Press, Cambridge, 2002)

2. A. Pelissetto, E. Vicari, Phys. Rep. 368, 549 (2002)

3. J.K. Bhattacharjee, U. Kaatze, S.Z. Mirzaev, Rep. Prog. Phys. 73, 066601 (2010)

4. R. Perl, R.A. Ferrell, Phys. Rev. Lett. 29, 51 (1972)

5. R. Perl, R.A. Ferrell, Phys. Rev. A 6, 2358 (1972)

6. T. Ohta, Prog. Theor. Phys. 54, 1566 (1975)

7. T. Ohta, K. Kawasaki, Prog. Theor. Phys. 55, 1384 (1976)

8. F. Garisto, R. Kapral, Phys. Rev. A 14, 884 (1976)

9. E.D. Siggia, B.I. Halperin, P.C. Hohenberg, Phys. Rev. B 13, 2110 (1976)

10. J. D. Gunton, in Dynamical Critical Phenomena and Related Topics, ed. by C. P. Enz (Springer, New York, 1979), p. 1

11. H.C. Burstyn, J.V. Sengers, P. Esfandiari, Phys. Rev. A 22, 282 (1980)

12. H.C. Burstyn, J.V. Sengers, Phys. Rev. Lett. 45, 259 (1980)

13. H.C. Burstyn, J.V. Sengers, Phys. Rev. A 25, 448 (1982)

14. R.F. Berg, M.R. Moldover, G.A. Zimmerli, Phys. Rev. Lett. 82, 920 (1999)

15. H. Hao, R.A. Ferrell, J.K. Bhattacharjee, Phys. Rev. E 71, 021201 (2005)

16. R.A. Ferrell, J.K. Bhattacharjee, Phys. Rev. B 24, 4095 (1981)

17. A.J. Lui, M.E. Fisher, Physica A 156, 35 (1989)

18. A.C. Flewelling, R.J. DeFonseka, N. Khaleeli, J. Partee, D.T. Jacobs, J. Chem. Phys. 104, 8048 (1996)

19. C.A. Cerdeiriña, J. Troncoso, E. Carballo, L. Romaní, Phys. Rev. E 66, 031507 (2002)

20. P.C. Hohenberg, B.I. Halperin, Rev. Mod. Phys. 49, 435 (1977)

21. L.I. Lisnyanskii, Y.S. Manucharov, I.G. Mikhailov, Sov. Phys. Acoust. 20, 566 (1975)

22. L.I. Lisnyanskii, Y.S. Manucharov, Sov. Phys. Acoust. 22, 33 (1976)

23. H. Tanaka, Y. Wada, H. Nakajima, Chem. Phys. 75, 37 (1983)

24. I. Iwanowski, R. Behrends, U. Kaatze, J. Chem. Phys. 120, 9192 (2004)

25. G. D’Arrigo, D. Sette, J. Chem. Phys. 48, 691 (1968)

26. S.S. Aliev, P.K. Khabibullaev, Sov. Phys. Acoust. 16, 108 (1970)

27. G. D’Arrigo, L. Mistura, P. Tartaglia, Phys. Rev. A 3, 1718 (1971)

28. N. Inoue, H. Takaoka, M. Kato, T. Hasegawa, K. Matsuzawa, Jpn. J. Appl. Phys. 30 (Suppl. 30-1), 25 (1991)

29. V.S. Sperkach, A.D. Alekhin, O.I. Bilous, Ukr. J. Phys. 49, 655 (2004) 
30. Y.S. Manucharov, I.G. Mikhailov, Sov. Phys. Acoust. 20, 176 (1974)

31. F. Eggers, U. Kaatze, K.H. Richmann, T. Telgmann, Meas. Sci. Technol. 5, 1131 (1994)

32. R.A. Ferrell, J.K. Bhattacharjee, Phys. Rev. A 31, 1788 (1985)

33. P. Debye, Polar Molecules (Chemical Catalog, New York, 1929)

34. S.Z. Mirzaev, U. Kaatze, Chem. Phys. 393, 129 (2012)

35. G. Zalczer, A. Bourgou, D. Beysens, Phys. Rev. A 28, 440 (1983)

36. M.L.S. Matos Lopes, C.A. Nieto de Castro, J.V. Sengers, Int. J. Thermophys. 13, 283 (1992)

37. I.R. Abdelraziq, S.S. Yun, F.B. Stumpf, J. Acoust. Soc. Am. 88, 1831 (1990)

38. H.C. Burstyn, J.V. Sengers, J.K. Bhattacharjee, R.A. Ferrell, Phys. Rev. A 28, 1567 (1983)

39. R. Behrends, I. Iwanowski, M. Kosmowska, A. Szala, U. Kaatze, J. Chem. Phys. 121, 5929 (2004)

40. S.-H. Chen, C.-C. Lai, J. Rouch, P. Tartaglia, Phys. Rev. A 27, 1086 (1983)

41. F. Eggers, U. Kaatze, Meas. Sci. Technol. 7, 1 (1996)

42. R. Polacek, U. Kaatze, Meas. Sci. Technol. 12, 1 (2001)

43. I. I. Shinder, V. N. Hudaiberdyev, L. A. Davydovich in Proceedings of the 25th Conferenc on Acoustics: Ultrasound-86, vol. 1 (Bratislava, 1986), p. 98

44. L.A. Davidovich, M.K. Karabaev, I.I. Shinder, Sov. Phys. Acoust. 36, 205 (1990)

45. L.A. Davidovich, I.I. Shinder, E.K. Kanaki, Phys. Rev. A 43, 1813 (1991)

46. G. Herzfeld, T. Litovitz, Absorption and Dispersion of Ultrasonic Waves (Academic, New York, 1959) 